\title{
Recent advances in the involvement of long non-coding RNAs in neural stem cell biology and brain pathophysiology
}

\section{Daphne Antoniou ${ }^{\dagger}$, Athanasios Stergiopoulos $^{\dagger}$ and Panagiotis K. Politis*}

Center for Basic Research, Biomedical Research Foundation of the Academy of Athens, Athens, Greece

\section{Edited by:}

Eumorphia Remboutsika, BSRC

"Alexander Fleming", Greece

\section{Reviewed by:}

Jack Price, National Institute for

Medical Research, UK

Benedikt Berninger, University

Medical Center of the Johannes

Gutenberg University Mainz,

Germany

Gianpaolo Papaccio, Second

University of Naples, Italy

Salvador Martinez, University

Miguel Hernandez, Spain

${ }^{*}$ Correspondence:

Panagiotis K. Politis, Center for Basic

Research, Biomedical Research

Foundation of the Academy of

Athens, 4 Soranou Efesiou Str., 115

27 Papagou, Athens, Greece

e-mail:ppolitis@bioacademy.gr

these authors have contributed

equally to this work.
Exploration of non-coding genome has recently uncovered a growing list of formerly unknown regulatory long non-coding RNAs (IncRNAs) with important functions in stem cell pluripotency, development and homeostasis of several tissues. Although thousands of IncRNAs are expressed in mammalian brain in a highly patterned manner, their roles in brain development have just begun to emerge. Recent data suggest key roles for these molecules in gene regulatory networks controlling neuronal and glial cell differentiation. Analysis of the genomic distribution of genes encoding for IncRNAs indicates a physical association of these regulatory RNAs with transcription factors (TFs) with well-established roles in neural differentiation, suggesting that IncRNAs and TFs may form coherent regulatory networks with important functions in neural stem cells (NSCs). Additionally, many studies show that IncRNAs are involved in the pathophysiology of brain-related diseases/disorders. Here we discuss these observations and investigate the links between IncRNAs, brain development and brain-related diseases. Understanding the functions of IncRNAs in NSCs and brain organogenesis could revolutionize the basic principles of developmental biology and neuroscience.

Keywords: non-coding genome, regulatory RNAs, gene regulatory networks, neural differentiation, neurogenesis, gliogenesis, brain-related diseases

\section{INTRODUCTION}

A long standing question in biological sciences is how cell diversity, specification patterns, and tissue complexity are generated during organ development. The mammalian brain is the most complex organ of any living organism. The question of how this enormous complexity is generated is still open. Such complexity is the result of millions of years of evolution that have equipped neural stem/progenitor cells (NSCs/NPCs) in the embryo with the ability to generate every neuron and glial cell in the brain via a combined action of extrinsic morphogenetic cues and intrinsic gene regulatory networks. For long time, it was thought that these networks are mainly based on cross-regulatory interactions between transcription factors (TFs) (Jessell, 2000; Politis et al., 2008; Kaltezioti et al., 2010; Martynoga et al., 2012; Stergiopoulos and Politis, 2013). However, the recent advent of sequencing

\footnotetext{
Abbreviations: lncRNA, long non-coding RNA; lincRNA, large intergenic noncoding RNA; miRNA, microRNA; NSCs, neural stem cells; ESCs, embryonic stem cells; iPSCs, induced pluripotent stem cells; CNS, central nervous system; SVZ, subventricular zone; ChIP, chromatin immunoprecipitation; RBP, RNA binding protein; RNA-Seq, RNA sequencing; RIP-Chip, RBP immunoprecipitation-microarray (Chip); ChIRP, chromatin isolation by RNA precipitation; CHART, capture hybridization analysis of RNA targets; CLIP, crosslinking-immunopurification; UCSC, University of California Santa Cruz; ORFs, open reading frames; TFs, transcription factors; HAR1, human accelerated region 1; AD, Alzheimer's disease; HD, Huntington's disease; DlxlAS, distal-less homeobox 1 antisense; Miat, myocardial infarction associated transcript; Rmst, rhabdomyosarcoma 2 associated transcript; Evf2, embryonic ventral forebrain 2; UtNgn1, untranslated neurogenin1; NEAT, non-coding nuclear enriched abundant transcript.
}

methodologies and experimental data from large-scale consortia focused on characterizing functional genomic elements such as ENCODE and FANTOM, have revolutionized our view for organization, activity, and regulation of the mammalian genome (Carninci et al., 2005; Katayama et al., 2005; Birney et al., 2007). Surprisingly, the vast majority of the genome is transcribed producing not only protein-coding RNAs but also a vast number of different kinds of newly-identified classes of non-coding RNAs, including microRNAs (miRNAs-small non-coding RNAs) and long non-coding RNAs (lncRNAs). miRNAs have been extensively studied while the role of lncRNAs in cell biology together with novel roles of miRNAs in directly regulating lncRNAs have just begun to emerge (Amodio et al., 2012a,b; Rossi et al., 2013; Tay et al., 2014). LncRNAs are transcribed by RNA polymerase II, defined as endogenous cellular RNAs longer than $200 \mathrm{nt}$ in length that lack an ORF, can be post-transcriptional processed by $5^{\prime}$ capping, polyadenylation, splicing, RNA editing, and exhibit specific sub-cellular localization (Qureshi and Mehler, 2012). Most importantly, it was recently shown that lncRNAs participate in the gene regulatory networks controlling embryonic stem cell (ESC) pluripotency and metastasis of cancer cells, as well as development and function of various tissues (Guttman et al., 2011; Gutschner and Diederichs, 2012; Qureshi and Mehler, 2012; Yang et al., 2013). Although lncRNAs are the most abundant classes of RNAs (Katayama et al., 2005; Birney et al., 2007; Kapranov et al., 2007b; Kapranov and St Laurent, 2012), they remain poorly characterized and their roles in brain development have just 
begun to arise. Notably, it has been proposed that the number of lncRNAs far exceeds the number of protein-coding mRNAs in the mammalian transcriptome (Carninci et al., 2005; Kapranov et al., 2010) and the vast majority of them appears to be expressed in adult brain in a highly specific manner (Qureshi and Mehler, 2012).

In the last few years, many groups have focused their efforts toward understanding the actions of lncRNAs in brain function and evolution. In this mini-review, we highlight the emerging evidence of the involvement of this new class of RNA molecules in NSC biology during development and adulthood, in health and disease. In particular, we summarize recent progress regarding the participation of lncRNAs in the regulatory networks that control the fine balance between proliferation and differentiation decisions of NSCs. The ability of lncRNAs to interact with both genomic loci and protein products of TF genes appears to endow them with a remarkable capacity to control NSC maintenance and differentiation, suggesting an undoubtedly important influence on the pathophysiology of several neurodegenerative disorders (Kapranov et al., 2007a,b; Guttman et al., 2009; Khalil et al., 2009; Orom et al., 2010; Bian and Sun, 2011; Qureshi and Mehler, 2012; Spadaro and Bredy, 2012; Ng et al., 2013).

\section{LncRNAs: NEW PLAYERS IN CELL BIOLOGY}

LncRNAs can be classified as bidirectional, intronic, intergenic, sense, antisense or $3^{\prime}$-UTR (untranslated region) transcripts with respect to nearby protein-coding genes (Mercer et al., 2009; Derrien et al., 2012). Some lncRNAs are exported from nucleus and perform important functions in the cytoplasm, but more often they are found in nucleus, particularly associated with chromatin (Saxena and Carninci, 2011). In more detail, these transcripts may be bound by proteins, other RNA molecules or even fulfil intrinsic catalytic functions (Fedor and Williamson, 2005; Ye, 2007; Guttman and Rinn, 2012). The vast majority of these transcripts are not translated, supporting a role as noncoding RNAs rather than protein precursors (Banfai et al., 2012; Derrien et al., 2012). Furthermore, lncRNAs are developmentally regulated, expressed in specific cell types, associated with chromatin signatures indicating transcriptional regulation, are under evolutionary constraint and associated with carcinogenesis and other diseases; all of which support meaningful roles of lncRNAs (Qureshi and Mehler, 2012; Fatica and Bozzoni, 2014). LncRNAs also participate in a striking diversity of cellular processes including transcriptional regulation, genomic imprinting, alternative splicing, mRNA stability, translational control, DNA damage response, cell cycle regulation and organelle biogenesis (Rinn and Chang, 2012). Interestingly, it seems that knock-down or over-expression of many of these lncRNAs produce phenotypes that are very well correlated with their aberrant expression in disease states (Qureshi et al., 2010; Gutschner and Diederichs, 2012; Qureshi and Mehler, 2012). Therefore, we believe that lncRNA transcripts would eventually occupy a central place in our understanding of the molecular mechanisms underlying human diseases.

Systematical profiling and comprehensive annotation of lncRNAs in various cell types can shed light on the functionalities of these novel regulators. LncRNA-protein interactions can be currently predicted, approached and analyzed by various research advanced tools and strategies apart from bioinformatics, including high-throughput analysis of lncRNA expression [microarrays, RNA sequencing [RNA-Seq] (Mortazavi et al., 2008; Wang et al., 2009; Ilott and Ponting, 2013), RNA CaptureSeq, iSeeRNA [support vector machine (SVM)-based classifier] (Sun et al., 2013), BRIC-Seq [50-bromo-uridine (BrU) immunoprecipitation chase-deep sequencing analysis] (Imamachi et al., 2013)], RIP [RNA-binding protein (RBP) immunoprecipitation] coupled with microarray and high-throughput sequencing (RIPChip/Seq respectively) (Zhao et al., 2010; Jain et al., 2011), ChIRP (chromatin isolation by RNA purification) and CHART (capture hybridization analysis of RNA targets-High-throughput finding of RBPs and DNAs) as well as CLIP (crosslinkingimmunopurification)-Seq, which provides transcriptome-wide coverage for mapping RBP-binding sites (Khalil et al., 2009; Wu et al., 2010; Chu et al., 2011; Zhu et al., 2013).

\section{LncRNAs IN NEURAL STEM CELLS}

These technologies have greatly helped the efforts to elucidate the role of lncRNAs in NSCs, neural differentiation, migration and maturation. Toward this goal, Ramos and colleagues identified and predicted regulatory roles for more than 12,000 novel lncRNAs (2,265 lncRNAs had proximal protein-coding gene neighbors) in the subventricular zone (SVZ) of adult mice. In FACS-sorted NSCs, they found a unique IncRNA expression pattern for each of the three stages of neurogenesis analyzed. Moreover, by using ChIP-Seq (chromatin immunoprecipitation-sequencing), lncRNAs were shown to be transcriptionally regulated in a manner analogous to mRNAs, enabling the prediction of lncRNAs that may function in the glialneuronal lineage specification of multipotent adult NSCs (Ramos et al., 2013; Wang et al., 2013). Further transcriptome-nextgeneration-sequencing revealed that lncRNA-mediated alternative splicing of cell fate determinants controls stem-cell commitment during neurogenesis. Specifically, Aprea and coworkers identified several genic and intergenic lncRNAs that are functionally involved in neurogenic commitment, including Cosl1, Btg2-AS1, Gm17566, Miat (Myocardial infarction associated transcript-Gomafu), Rmst (Rhabdomyosarcoma 2 associated transcript), Gm17566, Gm14207, Gm16758, 2610307P16Rik, AC102815.1, C230034O21Rik and 9930014A18Rik (Aprea et al., 2013). Accordingly, subsequent studies using high-throughput transcriptomic data (microarray platform) to examine lncRNA differential expression in NSCs, GABAergic neurons and oligodendrocytes, led to the identification of lncRNAs that are dynamically regulated during neural lineage specification, neuronal-glia fate switching and oligodendrocyte maturation [i.e., Dlx1AS (Distal-less homeobox 1 antisense), Evf2 (Embryonic ventral forebrain 2), Rmst, utNgn1 (untranslated Neurogenin1), MALAT1 (metastasis-associated lung adenocarcinoma transcript 1; also named NEAT2)] (Mercer et al., 2010; Qureshi and Mehler, 2012; Ng et al., 2013). ChIP-Seq approaches were also developed to generate and investigate genome-wide chromatin-state maps. These functional analyses predicted hypothetical roles for 150 mammalian lincRNAs (long intergenic non-coding RNAs) in a "guilt-by-association" manner in NSCs. 


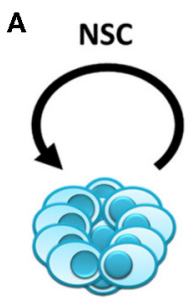

Self-renewal

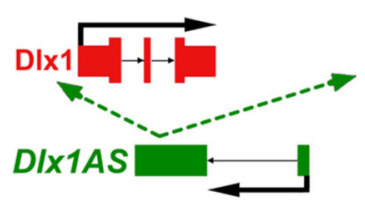

Differentiation

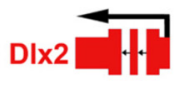

DIx2
$D / \times 1 / 2$

mRNA

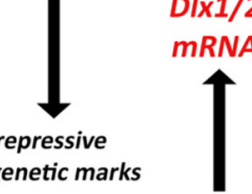

epigenetic mark

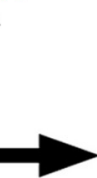

>ר

\section{Neurogenesis}

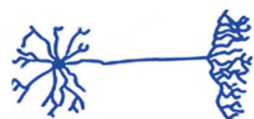

Ramos et al., 2013 Gonzales-Roybal and Lim, 2013
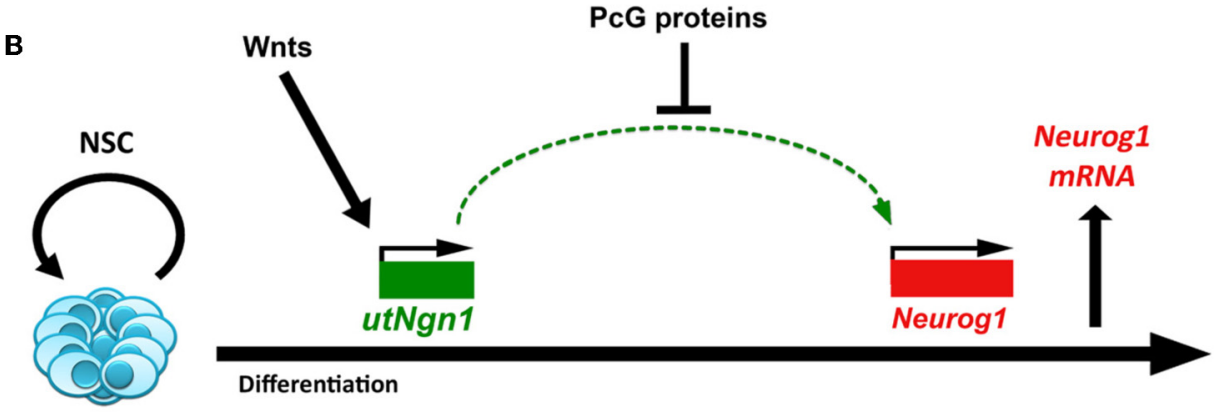

Neocortical

Neurogenesis

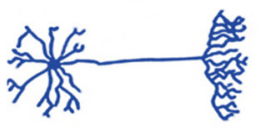

Self-renewal

Onoguchi et al., 2012

C

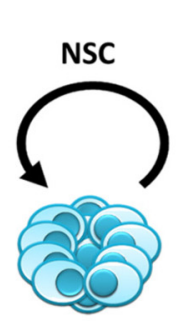

Self-renewal
Nkx2.2 isoform I

Nkx2.2 isoform II

Nkx2.2AS

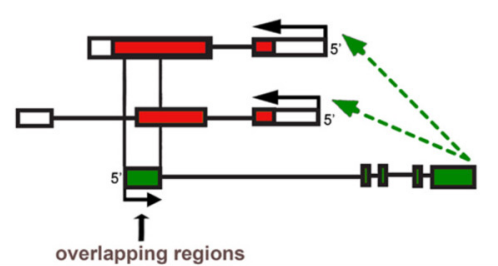

Differentiation
Nkx2.2

mRNA

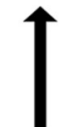

\section{Oligodendrogenesis}

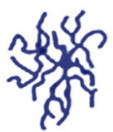

Tochitani and Hayashizaki, 2008

D

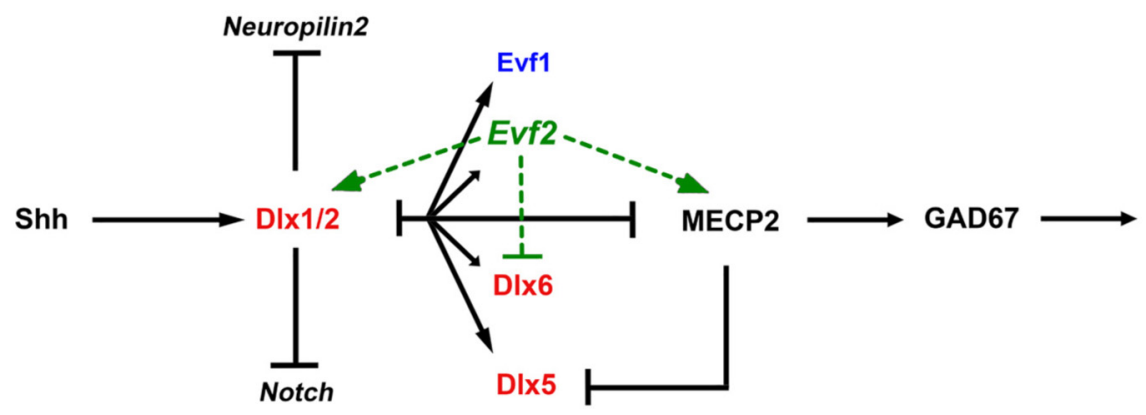

Forebrain Neurogenesis GABAergic interneurons

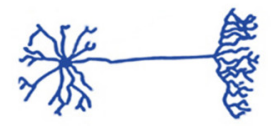

Bond et al., 2009

FIGURE 1 | Proposed schematic models for the role of different IncRNAs (green) in neural development. (A) LncRNA DIx1AS consists of two exons that are spliced and the mature transcript is polyadenylated. During neuronal differentiation of adult SVZ NSCs, DIX1AS is required for the induction of $D / x 1$ and D/x2 (red) gene expression (Gonzales-Roybal and Lim, 2013; Ramos et al., 2013). (B) During neocortical neurogenesis, utNgn1 receive Wnt signals (i.e., Wnt3a) to induce the expression of Neurog1 (red). PcG protein-mediated mechanisms (i.e., Ring1B, H3K27me3, H3K4me3, H3K9/K14ac) lead to the suppression of utNgn1 (Onoguchi et al., 2012). (C) The overlapping regions of $N k \times 2.2 A S$ and $N k \times 2.2$ isoforms (/ and /I-red) are necessary to promote $N k x 2.2$ mRNA levels and the subsequent oligodendrocytic differentiation of NSCs (Tochitani and Hayashizaki, 2008). (D) Mechanistic pathway for Evf2-dependent interactions crucial for forebrain development. Secreted Shh (Sonic hedgehog) promotes expression of D/x 1 and $D / x 2$ (red), which sequentially suppress Neuropilin2 and Notch signaling. Activation of Evf2 leads to the formation of a regulatory network together with D/x's (red) and MECP2 (methyl CpG binding protein 2) that controls GAD67 and GABAergic-interneuron formation (Bond et al., 2009). NSC, neural stem cell; Evf1 (IncRNA) (blue), Embryonic ventral forebrain-1, DIx6 antisense RNA 1 (DIx6AS1). 
In particular, specific lincRNAs appeared to localize near genes encoding key TFs (such as Sox2, Klf4, Myc, p53, NFkB and Brn1) that are involved in processes ranging from hippocampal development to neuronal and oligodendrocyte maturation (Guttman et al., 2009; Qureshi and Mehler, 2012).

More concisely, Ramos et al. have also performed in vitro knock-down studies and observed that Dlx1AS, a lncRNA encoded from a bigene $D l x 1 / 2$ cluster, is associated with fate determination of adult SVZ NSCs via positive regulation of $D l x 1$ and $D l x 2$ gene expression. Mechanistically, enhanced transcription of Dlx1AS occurs during neurogenesis when H3K27me3 (trimethylation of histone H3 Lys-27) repression is decreased. The H3K27me3-specific demethylase JMJD3 was also found to be enriched at the Dlx1AS locus (Figure 1A) (Gonzales-Roybal and Lim, 2013; Ramos et al., 2013). Recently, a comprehensive study came up with the finding that UtNgn1 is a non-coding RNA transcribed from an enhancer region of the Neurogenin1 (Neurog1) locus. This non-coding transcript seems to positively regulate Neurog1 transcription during neuronal differentiation of NSCs. In detail, during the late stage of neocortical NSC development, utNgn1 expression is up-regulated via involvement of Wnt signaling whereas it is down-regulated by PcG (polycomb group) proteins (Figure 1B) (Onoguchi et al., 2012). Additionally, two lncRNAs, nuclear enriched abundant transcripts NEAT1 and NEAT2 (MALAT1) are up-regulated in neuronal and glial progeny, and are associated with neuronal activity, growth and branching (Mercer et al., 2010; Ng et al., 2013). Miat (also called Gomafu) is one of the known non-coding RNAs that participates in neuronal subtype- specific determination and is also highly expressed in oligodendrocyte lineage specification (Aprea et al., 2013). In the same lineage, another lncRNA, $N k x 2.2 A S$, an antisense RNA of the known homeobox TF $N k x 2.2$, appears to lead NSCs into oligodendrocytic fate through modest induction of $N k \times 2.2$ gene. By using deletion mutants, Tochitani and Hayashizaki showed that the overlapping regions of $N k \times 2.2 A S$ and $N k \times 2.2$ isoforms are required for promoting $N k \times 2.2$ mRNA levels and the subsequent oligodendrocytic differentiation of NSCs (Figure 1C) (Tochitani and Hayashizaki, 2008). Evf2 is predominantly detected in the developing forebrain, both in human and mouse, and is critical for GABAergic-interneuron formation. Like other lncRNAs, Evf2 seems to control the expression of specific genes that are necessary during brain development, such as Dlx5,Dlx6 and Gad1, through distinct trans- and cis-acting mechanisms (Figure 1D) (Bond et al., 2009). Interestingly, Rmst is presented to be a novel marker for dopaminergic neurons during NSC differentiation, where it is co-expressed with the midbrain-specific TF Lmxla (Uhde et al., 2010).

Considering the tight connection between pluripotency of human ESCs and their differentiation into NSCs (ESC-derived NSCs) and subsequently into neurons, $\mathrm{Ng}$ and co-workers extensively showed that diverse lncRNAs are essential for ESC proliferation and neurogenesis through physical interaction with master TFs (e.g., Sox2). In detail, microarray data analysis led to the identification of 35 neuronal lncRNAs with important roles in neuronal differentiation, e.g., Rmst (AK056164, AF429305 and AF429306), IncRNA_N1 (AK124684), IncRNA_N2 (AK091713),
Table 1 | List of genes encoding TFs with critical roles in brain development that also contain IncRNA genes in close proximity to their genomic loci $(<3,000 \mathrm{bp})$.

\begin{tabular}{|c|c|c|}
\hline Genes encoding for TFs & LncRNAs & Position \\
\hline Ascl4 & AK018959 & Sense \\
\hline Atoh7 & AK005214 & Sense \\
\hline Crebbp & 4930455F16Rik & Intergenic \\
\hline Crx & CrxOS & Bidirectional \\
\hline Ctnnb1 & 4930593C16Rik & Bidirectional \\
\hline Cux2 & AK006762 & Sense \\
\hline Cux2 & AK187608 & Bidirectional \\
\hline Dlx1 & DIX1AS & Antisense \\
\hline Dlx4 & A730090H04Rik & Bidirectional \\
\hline Dlx6 & DIx6AS1 & Antisense \\
\hline Dlx6 & DIx6AS2 & Intronic \\
\hline Emx2 & $\mathrm{Em} \times 2 \mathrm{OS}$ & Bidirectional \\
\hline Evx1 & 5730457N03Rik & Bidirectional \\
\hline FezF1 & FezF1-AS1 & Bidirectional \\
\hline FezF1 & AK086573 & Intergenic \\
\hline FoxA2 & AK156045 & Antisense \\
\hline FoxG1 & AK158887 & Intergenic \\
\hline FoxG1 & 3110039M20Rik & Sense \\
\hline Gata1 & S57880 & Sense \\
\hline Gata2 & AK137172 & Sense \\
\hline Gata3 & 4930412013R & Bidirectional \\
\hline Gata4 & AK031341 & Intronic \\
\hline Gata6 & AK033147 & Bidirectional \\
\hline Gata6 & AK003136 & Bidirectional \\
\hline Gbx2 & D130058E05Rik & Bidirectional \\
\hline Gli1 & AK157048 & Sense \\
\hline Gli2 & AK054469 & Intronic \\
\hline Gli3 & AK135998 & Intergenic \\
\hline $\mathrm{Hm} \times 1$ & E130018015Rik & Bidirectional \\
\hline Irx2 & Gm20554 & Bidirectional \\
\hline Lef1 & Lef1-AS1 & Bidirectional \\
\hline Lhx1 & Lhx10S & Bidirectional \\
\hline Lhx3 & AK035055 & Bidirectional \\
\hline Lhx8 & Al606473 & Bidirectional \\
\hline$L m \times 1 b$ & C130021I20 & Bidirectional \\
\hline Lxrb (NR1H2) & AK184603 & $3^{\prime}-U T R$ \\
\hline Meis1 & AK144295 & Antisense \\
\hline Meis2 & AK012325 & Sense \\
\hline Meis2 & AK144367 & Bidirectional \\
\hline Meis2 & AK144485 & Bidirectional \\
\hline Msx1 & Msx1AS & Antisense \\
\hline MycN & MYCNOS & Bidirectional \\
\hline Myt1L & AK138505 & Antisense \\
\hline NFATc1 & AK155068 & $3^{\prime}-U T R$ \\
\hline NFATc4 & AK014164 & Sense \\
\hline NFIA & E130114P18Rik & Bidirectional \\
\hline NFIB & AK081607 & Intronic \\
\hline NFIx & AK168184 & Antisense \\
\hline NFkB2 & AK029443 & Bidirectional \\
\hline Ngn1 & AK016084 & Intergenic \\
\hline
\end{tabular}


Table 1 | Continued

\begin{tabular}{|c|c|c|}
\hline Genes encoding for TFs & LncRNAs & Position \\
\hline Nkx2.2 & $\mathrm{Nkx2.2AS}$ & Antisense \\
\hline Notch1 & AK075572 & Antisense \\
\hline NR2F1 & AK051417 & Intergenic \\
\hline NR2F1 & A830082K12Rik & Bidirectional \\
\hline NR2F2 & AK135306 & Intronic \\
\hline NR3C2 & Gm10649 & Bidirectional \\
\hline NR4A2 & BB557941 & Intergenic \\
\hline NR5A2 (Irh-1) & AK178198 & Antisense \\
\hline NR5A2 (Irh-1) & AK145521 & Intronic \\
\hline Otx2 & Otx2OS & Bidirectional \\
\hline Pax2 & AK006641 & Antisense \\
\hline Pax6 & AK044354 & Antisense \\
\hline $\mathrm{Pb} \times 3$ & AK138624 & Intronic \\
\hline Pou4F1 & AK084042 & Intergenic \\
\hline PPARd & AK033897 & Intronic \\
\hline PPARd & AK007468 & Intronic \\
\hline Prox1 & AK142161 & Bidirectional \\
\hline Ptf1a & AK053418 & Antisense \\
\hline RARa & AK031732 & Intronic \\
\hline $\mathrm{RARb}$ & AK052306 & $3^{\prime}-U T R$ \\
\hline RBPjK & AK164362 & Intronic \\
\hline Runx1 & AK131747 & Intergenic \\
\hline SATB2 & 9130024F11Rik & Bidirectional \\
\hline Six1 & AK035085 & $3^{\prime}-U T R$ \\
\hline Six3 & Six3OS1 & Bidirectional \\
\hline Six6 & 4930447C04Rik & Bidirectional \\
\hline Sox1 & Gm5607 & Sense \\
\hline Sox 10 & GM10863 & Bidirectional \\
\hline Sox2 & Sox2OT & Sense \\
\hline Sox21 & AK039417 & Bidirectional \\
\hline Sox8 & AK079380 & Bidirectional \\
\hline Sox9 & BC006965 & Bidirectional \\
\hline STAT5b & AK088966 & Intronic \\
\hline Tgif2 & 5430405H02Rik & $3^{\prime}-U T R$ \\
\hline THRA & AK165172 & Intronic \\
\hline THRB & AK088911 & Antisense \\
\hline WT1 & AK033304 & Intronic \\
\hline WT1 & Al314831 & Bidirectional \\
\hline Zeb1 & AK041408 & Intronic \\
\hline Zeb1 & Gm10125 & Bidirectional \\
\hline Zeb2 & Zeb2OS & Bidirectional \\
\hline
\end{tabular}

Some of these TFs contain more than one IncRNA near to their genes (duplicates). The position of each IncRNA relatively to its nearby protein-coding gene is indicated at the third column of the table.

IncRNA_N3 (AK055040, etc.). They also suggested that these lncRNAs are required for generation of neurons and suppression of gliogenesis by association with chromatin modifiers and nuclear proteins ( $\mathrm{Ng}$ et al., 2012). In agreement, another study that aimed at identifying functional features of $\operatorname{lncR}$ NAs during ESC differentiation to adult cerebellum by utilizing RNA-Seq and ChIP-Seq technologies, demonstrated that filtered novel lncRNAs are located close to protein-coding genes involved in functions such as neuronal differentiation and transcriptional regulation (Guttman et al., 2009; Lv et al., 2013). Particular studies have been successful in identifying anti-NOS2A as an antisense transcript of the NOS2A (Nitric oxide synthase 2 enzyme) gene, an isoform of the NOS protein which induces hESC differentiation into neurogenic precursors (Korneev et al., 2008). Specific expression pattern was also detected for Otx2c (Orthodenticle homeobox $2 \mathrm{c}$ ), an alternative splicing variant of the pre-mRNA Otx2 with a possible role in neural differentiation of hESCs (Liu et al., 2013). Another lncRNA that is correlated with the proliferation state of ESCs is lincRNA-Sox2, which is located at the promoter of Sox2 locus and is regulated by Sox2 and Oct4 (Guttman et al., 2009). Finally, a thorough targeted RNA-Seq analysis carried out using neurons derived from patient-specific induced pluripotent stem cells (iPSCs) showed that more than 1,500 lncRNAs are dynamically regulated during differentiation of iPSCs toward glutamatergic neurons. Particularly, the expression of 1,622 non-coding genes (lncRNAs/lincRNAs) was dramatically affected during conversion from iPSCs to differentiating neurons, while alternative splicing occurred. Significant alterations were also observed in the expression patterns of non-coding genes involved in neuropsychiatric disorders (Lin et al., 2011; Qureshi and Mehler, 2012; Akula et al., 2014).

\section{LnCRNAs IN BRAIN FUNCTION, EVOLUTION AND NEUROLOGICAL DISEASES}

The importance of lncRNAs in the brain is explicitly highlighted by the observation that most of them are expressed in the adult mammalian brain in highly regional-, cellular- and sub-cellular compartment-specific and neuronal activity dependent profiles (Mercer et al., 2008; Ponjavic et al., 2009; Qureshi and Mehler, 2012). In fact, it seems that lncRNAs have played important roles in the evolution of the form and function of human brain, since the fastest evolving regions of the primate genome are non-coding sequences that generate lncRNAs implicated in the modulation of neuro-developmental genes (Pollard et al., 2006; Qureshi and Mehler, 2012). The most dramatic of these regions, called HAR1 (human accelerated region 1), is part of a novel lncRNA gene $(H A R 1 F)$ that is expressed specifically in Cajal-Retzius neurons in the developing human neocortex from 7 to 19 gestational weeks, a crucial period for cortical neuron-specification and migration. Additionally, analysis of highly conserved lncRNAs in birds, marsupials and eutherian mammals revealed a remarkable similarity in spatiotemporal expression profiles of orthologous lncRNAs, suggesting ancient roles during brain development (Chodroff et al., 2010). Moreover, lncRNAs directly modulate synaptic processes (MALAT1), protein synthesis $(B C 1)$, learning and memory $(T s x)$, and are associated with neuronal activity in mouse cortex (Qureshi and Mehler, 2012). In agreement with these effects on neurons, lncRNAs have also been implicated in the pathophysiology of neuro-developmental, neurodegenerative, neuro-immunological and neuro-oncological diseases/disorders. For instance, high levels of $B C 1 / B C 200$ and $B A C E 1-A S$ have been implicated in Alzheimer's disease (AD) while NEAT1's in Huntington's disease (HD). BC1/BC200 normally seems to selectively regulate local protein synthesis in post-synaptic dendritic compartments, by repressing translation via an elF4- $A$ dependent mechanism (Qureshi et al., 2010; Niland et al., 2012). 


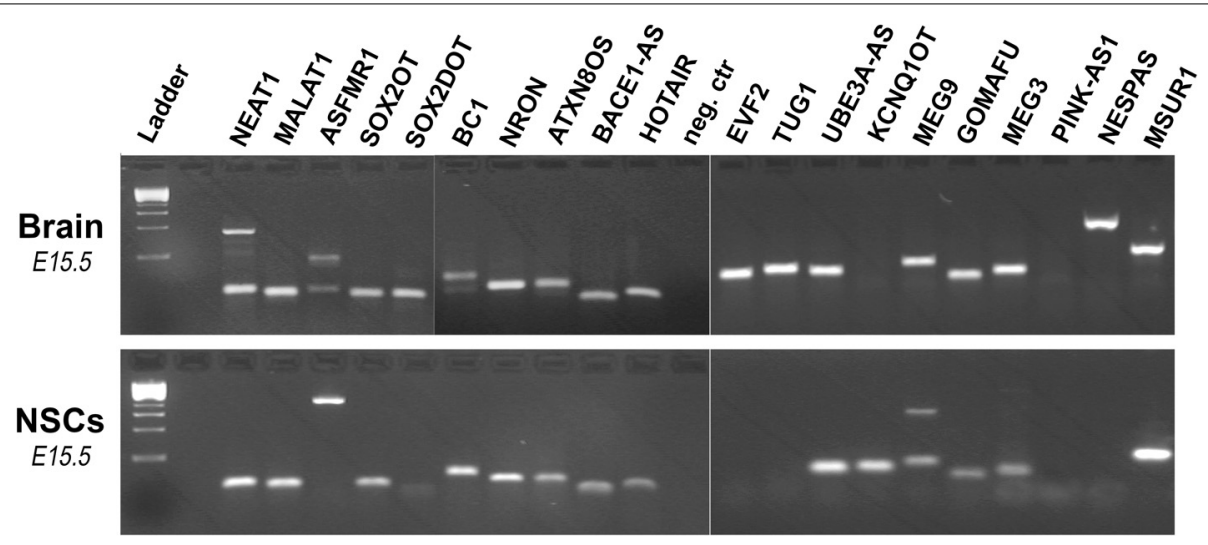

FIGURE 2 | RT-PCR-based detection of various IncRNAs associated with CNS-related diseases or disorders. Experiments were performed using cDNA samples derived from embryonic mouse brain (E15.5) or NSCs isolated from embryonic mouse telencephalon and cultured ex vivo, as indicated. In some cases alternative splicing isoforms are also evident. RT-PCR primer sequences are available upon request.
De-regulation of $B A C E 1-A S$ has been shown to be responsible for feed-forward induction of BACE1 ( $\beta$-secretase); thus leading to amyloid $\beta(A \beta)$ increased production and possible AD pathogenesis (Qureshi et al., 2010). Additionally, NEAT1 is a lncRNA known to be involved in cell death mechanisms. In particular, NEAT1 controls target gene transcription by protein sequestration into paraspeckles (stress-responsive sub-nuclear structures), a potentially dysfunctional pathway in HD progression. In accordance, Hirose and co-workers have very recently shown that NEAT1 transcriptional up-regulation leads to the enlargement of these structures after proteasome inhibition (Johnson, 2012; Hirose et al., 2014). Various lncRNAs have also been correlated with Parkinson's disease (PINK-AS1, UCHL1-AS1), amyotrophic lateral sclerosis (MSUR1), Down's syndrome (NRON) as well as with tumor progression (ANRIL, MALAT1, HOTAIR, NOS3AS) (Gutschner and Diederichs, 2012). A common theme emerging from all these cases is that lncRNAs control the expression of nearby protein-coding genes in cis and de-regulation of this relationship could lead to nervous system-related diseases. In agreement, brain-expressed lncRNAs are preferentially located adjacent to protein-coding genes that are also expressed in the brain and involved in transcriptional regulation and/or nervous system development (Mercer et al., 2008; Ponjavic et al., 2009). Many of these pairs often exhibited coordinated expression during developmental transitions. Therefore, it was suggested that these lncRNAs may influence the expression of the associated protein-coding genes similarly to previously characterized examples such as Nkx2.2AS, HOTAIR, p15AS, p21AS and Evf2.

\section{PERSPECTIVE LINKS BETWEEN IncRNAs AND BRAIN DEVELOPMENT}

Over recent years, it has become more and more evident that lncRNAs play key roles in gene regulatory networks controlling nervous system development. To initially investigate this link, we bioinformatically screened numerous mouse genes encoding TFs with well-established roles in CNS (central nervous system) development for close proximity with lncRNA genes (based on publicly available data from UCSC genome-browser).
Accordingly, we managed to identify 79 such TF genes encompassing 91 transcriptional units for lncRNA genes in close proximity ( $<3,000$ base pairs-bp) (Table 1 ). In particular, $40.65 \%$ of these lncRNAs are bidirectional, $16.48 \%$ intronic, $9.89 \%$ intergenic, $12.08 \%$ sense, $15.38 \%$ antisense and $5.49 \% 3^{\prime}$-UTR. The majority of these lncRNAs have only been identified in genomewide expression screens, are expressed in nervous system, and intriguingly, their functions are totally unknown. Some of them have been reported to interact with TFs or chromatin-associated complexes (Wang and Chang, 2011; Guttman and Rinn, 2012; Rinn and Chang, 2012), indicating potential contribution to the combinatorial transcriptional codes involved in NSC maintenance, sub-type/lineage specification and terminal differentiation. Based on these preliminary evidence, we postulate that lncRNAs and TFs with regulatory role in neurogenesis are interconnected and may form coherent cross-regulatory networks that are associated with CNS development. However, this hypothesis needs to be extensively interrogated in NSCs. Moreover, considering the fact that some TFs are used as reprogramming tools for the production of iPSCs, induced neurons, induced NSCs, cardiomyocytes, etc. (Yang et al., 2011), these lncRNAs may be utilized for the production of clinically useful cell types in the near feature, revolutionizing the basic principles of developmental/cell biology as well as neuroscience.

To further examine the links between lncRNAs, brain development and neurological disorders, we tested whether lncRNAs, associated with nervous system-related diseases in humans, are expressed in embryonic mouse brain (E15.5) or ex vivo cultured NSCs (isolated from mouse telencephalon, E15.5). The rational for these experiments was based on observations showing many neurological diseases to be initiated very early during brain maturation and development (Bian and Sun, 2011; Qureshi and Mehler, 2012). Specifically, we first identified in silico and then tested for expression 20 such lncRNAs with clear conservation in mouse genome. Interestingly, RT-PCR (reverse transcriptionPCR) analysis revealed that most lncRNAs are expressed in both brain and NSCs (Figure 2), suggesting potential involvement in brain development. Moreover, a possible function of these 
lncRNAs in NSCs may contribute to their roles in neurological diseases. Nevertheless, further investigation and initiation of new experimental studies are needed to uncover the exact function of lncRNAs in NSCs and nervous system in general.

\section{CONCLUSIONS}

LncRNAs represent a new exciting frontier in molecular biology with major roles in NSC fate decisions, specification and commitment. Full understanding of how these non-coding transcripts regulate the expression of protein-coding genes and participate in gene regulatory circuitries could lead to the discovery of novel cellular/molecular mechanisms and signaling pathways involved in neural development; thus having potential implications for the treatment of nervous system-related diseases and traumas.

\section{ACKNOWLEDGMENTS}

This work was supported by ARISTEIA-II (NeuroNetwk, No. 4786), IKYDA (Greek Ministry of Education) and Fondation Santé grants to Panagiotis K. Politis.

\section{REFERENCES}

Akula, N., Barb, J., Jiang, X., Wendland, J. R., Choi, K. H., Sen, S. K., et al. (2014). RNA-sequencing of the brain transcriptome implicates dysregulation of neuroplasticity, circadian rhythms and GTPase binding in bipolar disorder. Mol. Psychiatry. doi: 10.1038/mp.2013.170. [Epub ahead of print].

Amodio, N., Di Martino, M. T., Foresta, U., Leone, E., Lionetti, M., Leotta, M., et al. (2012a). miR-29b sensitizes multiple myeloma cells to bortezomib-induced apoptosis through the activation of a feedback loop with the transcription factor Sp1. Cell Death Dis. 3, e436. doi: 10.1038/cddis.2012.175

Amodio, N., Leotta, M., Bellizzi, D., Di Martino, M. T., D’Aquila, P., Lionetti, M., et al. (2012b). DNA-demethylating and anti-tumor activity of synthetic miR29b mimics in multiple myeloma. Oncotarget 3, 1246-1258.

Aprea, J., Prenninger, S., Dori, M., Ghosh, T., Monasor, L. S., Wessendorf, E., et al. (2013). Transcriptome sequencing during mouse brain development identifies long non-coding RNAs functionally involved in neurogenic commitment. EMBO J. 32, 3145-3160. doi: 10.1038/emboj.2013.245

Banfai, B., Jia, H., Khatun, J., Wood, E., Risk, B., Gundling, W. E., et al. (2012). Long noncoding RNAs are rarely translated in two human cell lines. Genome Res. 22, 1646-1657. doi: 10.1101/gr.134767.111

Bian, S., and Sun, T. (2011). Functions of noncoding RNAs in neural development and neurological diseases. Mol. Neurobiol. 44, 359-373. doi: 10.1007/s12035011-8211-3

Birney, E., Stamatoyannopoulos, J. A., Dutta, A., Guigo, R., Gingeras, T. R., Margulies, E. H., et al. (2007). Identification and analysis of functional elements in $1 \%$ of the human genome by the ENCODE pilot project. Nature 447 , 799-816. doi: 10.1038/nature05874

Bond, A. M., Vangompel, M. J., Sametsky, E. A., Clark, M. F., Savage, J. C., Disterhoft, J. F., et al. (2009). Balanced gene regulation by an embryonic brain ncRNA is critical for adult hippocampal GABA circuitry. Nat. Neurosci. 12, 1020-1027. doi: 10.1038/nn.2371

Carninci, P., Kasukawa, T., Katayama, S., Gough, J., Frith, M. C., Maeda, N., et al. (2005). The transcriptional landscape of the mammalian genome. Science 309, 1559-1563. doi: 10.1126/science.1112014

Chodroff, R. A., Goodstadt, L., Sirey, T. M., Oliver, P. L., Davies, K. E., Green, E. D., et al. (2010). Long noncoding RNA genes: conservation of sequence and brain expression among diverse amniotes. Genome Biol. 11:R72. doi: 10.1186/gb-2010-11-7-r72

Chu, C., Qu, K., Zhong, F. L., Artandi, S. E., and Chang, H. Y. (2011). Genomic maps of long noncoding RNA occupancy reveal principles of RNA-chromatin interactions. Mol. Cell 44, 667-678. doi: 10.1016/j.molcel.2011.08.027

Derrien, T., Johnson, R., Bussotti, G., Tanzer, A., Djebali, S., Tilgner, H., et al. (2012). The GENCODE v7 catalog of human long noncoding RNAs: analysis of their gene structure, evolution, and expression. Genome Res. 22, 1775-1789. doi: 10.1101/gr.132159.111
Fatica, A., and Bozzoni, I. (2014). Long non-coding RNAs: new players in cell differentiation and development. Nat. Rev. Genet. 15, 7-21. doi: 10.1038/nrg3606

Fedor, M. J., and Williamson, J. R. (2005). The catalytic diversity of RNAs. Nat. Rev. Mol. Cell Biol. 6, 399-412. doi: 10.1038/nrm1647

Gonzales-Roybal, G., and Lim, D. A. (2013). Chromatin-based epigenetics of adult subventricular zone neural stem cells. Front. Genet. 4:194. doi: 10.3389/fgene.2013.00194

Gutschner, T., and Diederichs, S. (2012). The hallmarks of cancer: a long noncoding RNA point of view. RNA Biol. 9, 703-719. doi: 10.4161/rna.20481

Guttman, M., Amit, I., Garber, M., French, C., Lin, M. F., Feldser, D., et al. (2009). Chromatin signature reveals over a thousand highly conserved large non-coding RNAs in mammals. Nature 458, 223-227. doi: 10.1038/nature07672

Guttman, M., Donaghey, J., Carey, B. W., Garber, M., Grenier, J. K., Munson, G., et al. (2011). lincRNAs act in the circuitry controlling pluripotency and differentiation. Nature 477, 295-300. doi: 10.1038/nature10398

Guttman, M., and Rinn, J. L. (2012). Modular regulatory principles of large noncoding RNAs. Nature 482, 339-346. doi: 10.1038/nature10887

Hirose, T., Virnicchi, G., Tanigawa, A., Naganuma, T., Li, R., Kimura, H., et al. (2014). NEAT1 long noncoding RNA regulates transcription via protein sequestration within subnuclear bodies. Mol. Biol. Cell 25, 169-183. doi: 10.1091/mbc.E13-09-0558

Ilott, N. E., and Ponting, C. P. (2013). Predicting long non-coding RNAs using RNA sequencing. Methods 63, 50-59. doi: 10.1016/j.ymeth.2013.03.019

Imamachi, N., Tani, H., Mizutani, R., Imamura, K., Irie, T., Suzuki, Y., et al. (2013). BRIC-seq: a genome-wide approach for determining RNA stability in mammalian cells. Methods. doi: 10.1016/j.ymeth.2013.07.014. [Epub ahead of print].

Jain, R., Devine, T., George, A. D., Chittur, S. V., Baroni, T. E., Penalva, L. O., et al. (2011). RIP-Chip analysis: RNA-Binding Protein ImmunoprecipitationMicroarray (Chip) Profiling. Methods Mol. Biol. 703, 247-263. doi: 10.1007/9781-59745-248-9_17

Jessell, T. M. (2000). Neuronal specification in the spinal cord: inductive signals and transcriptional codes. Nat. Rev. Genet. 1, 20-29. doi: 10.1038/35049541

Johnson, R. (2012). Long non-coding RNAs in Huntington's disease neurodegeneration. Neurobiol. Dis. 46, 245-254. doi: 10.1016/j.nbd.2011.12.006

Kaltezioti, V., Kouroupi, G., Oikonomaki, M., Mantouvalou, E., Stergiopoulos, A., Charonis, A., et al. (2010). Prox1 regulates the notch1-mediated inhibition of neurogenesis. PLoS Biol. 8:e1000565. doi: 10.1371/journal.pbio.1000565

Kapranov, P., Cheng, J., Dike, S., Nix, D. A., Duttagupta, R., Willingham, A. T., et al. (2007a). RNA maps reveal new RNA classes and a possible function for pervasive transcription. Science 316, 1484-1488. doi: 10.1126/science.1138341

Kapranov, P., and St Laurent, G. (2012). Dark matter RNA: existence, function, and controversy. Front. Genet. 3:60. doi: 10.3389/fgene.2012.00060

Kapranov, P., St Laurent, G., Raz, T., Ozsolak, F., Reynolds, C. P., Sorensen, P. H., et al. (2010). The majority of total nuclear-encoded non-ribosomal RNA in a human cell is "dark matter" un-annotated RNA. BMC Biol. 8:149. doi: 10.1186/1741-7007-8-149

Kapranov, P., Willingham, A. T., and Gingeras, T. R. (2007b). Genome-wide transcription and the implications for genomic organization. Nat. Rev. Genet. 8, 413-423. doi: 10.1038/nrg2083

Katayama, S., Tomaru, Y., Kasukawa, T., Waki, K., Nakanishi, M., Nakamura, M., et al. (2005). Antisense transcription in the mammalian transcriptome. Science 309, 1564-1566. doi: 10.1126/science.1112009

Khalil, A. M., Guttman, M., Huarte, M., Garber, M., Raj, A., Rivea Morales, D., et al. (2009). Many human large intergenic noncoding RNAs associate with chromatin-modifying complexes and affect gene expression. Proc. Natl. Acad. Sci. U.S.A. 106, 11667-11672. doi: 10.1073/pnas.0904715106

Korneev, S. A., Korneeva, E. I., Lagarkova, M. A., Kiselev, S. L., Critchley, G., and O'Shea, M. (2008). Novel noncoding antisense RNA transcribed from human anti-NOS2A locus is differentially regulated during neuronal differentiation of embryonic stem cells. RNA 14, 2030-2037. doi: 10.1261/rna 1084308

Lin, M., Pedrosa, E., Shah, A., Hrabovsky, A., Maqbool, S., Zheng, D., et al. (2011). RNA-Seq of human neurons derived from iPS cells reveals candidate long noncoding RNAs involved in neurogenesis and neuropsychiatric disorders. PLoS ONE 6:e23356. doi: 10.1371/journal.pone.0023356

Liu, Z., Chi, L., Fang, Y., Liu, L., and Zhang, X. (2013). Specific expression pattern of a novel Otx2 splicing variant during neural differentiation. Gene 523, 33-38. doi: 10.1016/j.gene.2013.03.114 
Lv, J., Liu, H., Huang, Z., Su, J., He, H., Xiu, Y., et al. (2013). Long non-coding RNA identification over mouse brain development by integrative modeling of chromatin and genomic features. Nucleic Acids Res. 41, 10044-10061. doi: 10.1093/nar/gkt818

Martynoga, B., Drechsel, D., and Guillemot, F. (2012). Molecular control of neurogenesis: a view from the mammalian cerebral cortex. Cold Spring Harb. Perspect. Biol. 4:a008359. doi: 10.1101/cshperspect.a008359

Mercer, T. R., Dinger, M. E., and Mattick, J. S. (2009). Long non-coding RNAs: insights into functions. Nat. Rev. Genet. 10, 155-159. doi: 10.1038/nrg2521

Mercer, T. R., Dinger, M. E., Sunkin, S. M., Mehler, M. F., and Mattick, J. S. (2008). Specific expression of long noncoding RNAs in the mouse brain. Proc. Natl. Acad. Sci. U.S.A. 105, 716-721. doi: 10.1073/pnas.0706729105

Mercer, T. R., Qureshi, I. A., Gokhan, S., Dinger, M. E., Li, G., Mattick, J. S. et al. (2010). Long noncoding RNAs in neuronal-glial fate specification and oligodendrocyte lineage maturation. BMC Neurosci. 11:14. doi: 10.1186/14712202-11-14

Mortazavi, A., Williams, B. A., McCue, K., Schaeffer, L., and Wold, B. (2008). Mapping and quantifying mammalian transcriptomes by RNA-Seq. Nat. Methods 5, 621-628. doi: 10.1038/nmeth.1226

Ng, S. Y., Johnson, R., and Stanton, L. W. (2012). Human long non-coding RNAs promote pluripotency and neuronal differentiation by association with chromatin modifiers and transcription factors. EMBO J. 31, 522-533. doi: 10.1038/emboj.2011.459

Ng, S. Y., Lin, L., Soh, B. S., and Stanton, L. W. (2013). Long noncoding RNAs in development and disease of the central nervous system. Trends Genet. 29, 461-468. doi: 10.1016/j.tig.2013.03.002

Niland, C. N., Merry, C. R., and Khalil, A. M. (2012). Emerging roles for long non-coding rnas in cancer and neurological disorders. Front. Genet. 3:25. doi: 10.3389/fgene.2012.00025

Onoguchi, M., Hirabayashi, Y., Koseki, H., and Gotoh, Y. (2012). A noncoding RNA regulates the neurogenin 1 gene locus during mouse neocortical development. Proc. Natl. Acad. Sci. U.S.A. 109, 16939-16944. doi: 10.1073/pnas.1202956109

Orom, U. A., Derrien, T., Beringer, M., Gumireddy, K., Gardini, A., Bussotti, G. et al. (2010). Long noncoding RNAs with enhancer-like function in human cells. Cell 143, 46-58. doi: 10.1016/j.cell.2010.09.001

Politis, P. K., Thomaidou, D., and Matsas, R. (2008). Coordination of cell cycle exit and differentiation of neuronal progenitors. Cell Cycle 7, 691-697. doi: $10.4161 /$ cc.7.6.5550

Pollard, K. S., Salama, S. R., Lambert, N., Lambot, M. A., Coppens, S., Pedersen, J. S., et al. (2006). An RNA gene expressed during cortical development evolved rapidly in humans. Nature 443, 167-172. doi: 10.1038/nature05113

Ponjavic, J., Oliver, P. L., Lunter, G., and Ponting, C. P. (2009). Genomic and transcriptional co-localization of protein-coding and long non-coding RNA pairs in the developing brain. PLoS Genet. 5:e1000617. doi: 10.1371/journal.pgen.1000617

Qureshi, I. A., Mattick, J. S., and Mehler, M. F. (2010). Long non-coding RNAs in nervous system function and disease. Brain Res. 1338, 20-35. doi: 10.1016/j.brainres.2010.03.110

Qureshi, I. A., and Mehler, M. F. (2012). Emerging roles of non-coding RNAs in brain evolution, development, plasticity and disease. Nat. Rev. Neurosci. 13 , 528-541. doi: 10.1038/nrn3234

Ramos, A. D., Diaz, A., Nellore, A., Delgado, R. N., Park, K. Y., Gonzales-Roybal, G., et al. (2013). Integration of genome-wide approaches identifies lncRNAs of adult neural stem cells and their progeny in vivo. Cell Stem Cell 12, 616-628. doi: 10.1016/j.stem.2013.03.003

Rinn, J. L., and Chang, H. Y. (2012). Genome regulation by long noncoding RNAs. Annu. Rev. Biochem. 81, 145-166. doi: 10.1146/annurev-biochem-051410092902

Rossi, M., Pitari, M. R., Amodio, N., Di Martino, M. T., Conforti, F., Leone, E., et al. (2013). miR-29b negatively regulates human osteoclastic cell differentiation and function: implications for the treatment of multiple myeloma-related bone disease. J. Cell. Physiol. 228, 1506-1515. doi: 10.1002/jcp.24306
Saxena, A., and Carninci, P. (2011). Long non-coding RNA modifies chromatin: epigenetic silencing by long non-coding RNAs. Bioessays 33, 830-839. doi: 10.1002/bies.201100084

Spadaro, P. A., and Bredy, T. W. (2012). Emerging role of non-coding RNA in neural plasticity, cognitive function, and neuropsychiatric disorders. Front. Genet. 3:132. doi: 10.3389/fgene.2012.00132

Stergiopoulos, A., and Politis, P. K. (2013). The role of nuclear receptors in controlling the fine balance between proliferation and differentiation of neural stem cells. Arch. Biochem. Biophys. 534, 27-37. doi: 10.1016/j.abb.2012.09.009

Sun, K., Chen, X., Jiang, P., Song, X., Wang, H., and Sun, H. (2013). iSeeRNA: identification of long intergenic non-coding RNA transcripts from transcriptome sequencing data. BMC Genomics 14(Suppl. 2):S7. doi: 10.1186/1471-216414-S2-S7

Tay, Y., Rinn, J., and Pandolfi, P. P. (2014). The multilayered complexity of ceRNA crosstalk and competition. Nature 505, 344-352. doi: 10.1038/nature12986

Tochitani, S., and Hayashizaki, Y. (2008). Nkx2.2 antisense RNA overexpression enhanced oligodendrocytic differentiation. Biochem. Biophys. Res. Commun. 372, 691-696. doi: 10.1016/j.bbrc.2008.05.127

Uhde, C. W., Vives, J., Jaeger, I., and Li, M. (2010). Rmst is a novel marker for the mouse ventral mesencephalic floor plate and the anterior dorsal midline cells. PLoS ONE 5:e8641. doi: 10.1371/journal.pone.0008641

Wang, J., Lucas, B. A., and Maquat, L. E. (2013). New gene expression pipelines gush lncRNAs. Genome Biol. 14:117. doi: 10.1186/gb-2013-14-5-117

Wang, K. C., and Chang, H. Y. (2011). Molecular mechanisms of long noncoding RNAs. Mol. Cell 43, 904-914. doi: 10.1016/j.molcel.2011.08.018

Wang, Z., Gerstein, M., and Snyder, M. (2009). RNA-Seq: a revolutionary tool for transcriptomics. Nat. Rev. Genet. 10, 57-63. doi: 10.1038/nrg2484

Wu, J. Q., Habegger, L., Noisa, P., Szekely, A., Qiu, C., Hutchison, S., et al. (2010) Dynamic transcriptomes during neural differentiation of human embryonic stem cells revealed by short, long, and paired-end sequencing. Proc. Natl. Acad. Sci. U.S.A. 107, 5254-5259. doi: 10.1073/pnas.0914114107

Yang, L., Lin, C., Jin, C., Yang, J. C., Tanasa, B., Li, W., et al. (2013). IncRNAdependent mechanisms of androgen-receptor-regulated gene activation programs. Nature 500, 598-602. doi: 10.1038/nature 12451

Yang, N., Ng, Y. H., Pang, Z. P., Sudhof, T. C., and Wernig, M. (2011). Induced neuronal cells: how to make and define a neuron. Cell Stem Cell 9, 517-525. doi: 10.1016/j.stem.2011.11.015

Ye, K. (2007). H/ACA guide RNAs, proteins and complexes. Curr. Opin. Struct. Biol. 17, 287-292. doi: 10.1016/j.sbi.2007.05.012

Zhao, J., Ohsumi, T. K., Kung, J. T., Ogawa, Y., Grau, D. J., Sarma, K., et al. (2010) Genome-wide identification of polycomb-associated RNAs by RIP-seq. Mol. Cell 40, 939-953. doi: 10.1016/j.molcel.2010.12.011

Zhu, J., Fu, H., Wu, Y., and Zheng, X. (2013). Function of IncRNAs and approaches to lncRNA-protein interactions. Sci. China Life Sci. 56, 876-885. doi: $10.1007 / \mathrm{s} 11427-013-4553-6$

Conflict of Interest Statement: The authors declare that the research was conducted in the absence of any commercial or financial relationships that could be construed as a potential conflict of interest.

Received: 31 January 2014; accepted: 02 April 2014; published online: 22 April 2014. Citation: Antoniou D, Stergiopoulos A and Politis PK (2014) Recent advances in the involvement of long non-coding RNAs in neural stem cell biology and brain pathophysiology. Front. Physiol. 5:155. doi: 10.3389/fphys.2014.00155

This article was submitted to Craniofacial Biology, a section of the journal Frontiers in Physiology.

Copyright (c) 2014 Antoniou, Stergiopoulos and Politis. This is an open-access article distributed under the terms of the Creative Commons Attribution License (CC BY). The use, distribution or reproduction in other forums is permitted, provided the original author(s) or licensor are credited and that the original publication in this journal is cited, in accordance with accepted academic practice. No use, distribution or reproduction is permitted which does not comply with these terms. 